\title{
A LINEAR TIME ALGORITHM FOR NEAR MINIMAX CONTINUOUS PIECEWISE LINEAR REPRESENTATIONS OF DISCRETE DATA*
}

\author{
EMILY K. SZUSZ ${ }^{\dagger}$ AND ALLAN R. WILLMS ${ }^{\dagger}$
}

\begin{abstract}
An algorithm that constructs a continuous piecewise linear representation, subject to a certain slope/segment length constraint, of a given set of data is described. The algorithm yields an optimal or near optimal representation, subject to this constraint, in the $L_{\infty}$ norm and does so in at worst $O(n K)$ time, where $n$ is the number of data points and $K$ the number of segments. The constraint is determined by a user-specified parameter, $t_{\min }$, which dictates a lower bound for the distance between opposite-sign slope discontinuities. For reasonable $t_{\min }$ values, the resulting representation of the data captures the signal and both smooths the noise and provides a measure of it. This representation is useful for applications that require a specified interval for the data values and also allows easy and continuous interpolation of ranges between recorded time points. The algorithm is described, some of its properties are proven, and its capabilities demonstrated with several examples. Comparisons are made with alternative techniques.
\end{abstract}

Key words. minimax approximation, piecewise linear representations, Remez algorithm, parameter estimation

AMS subject classifications. 65D10, 65G40

DOI. $10.1137 / 090769077$

1. Introduction. Consider the problem of finding alternate representations of discrete time series data. The effectiveness of a particular representation depends on its end uses, but nearly all representations offer a degree of compactification, capture important informational features of the data, allow for interpolation of values between recorded points, and are easier to work with than the raw data. In applications such as pattern recognition [19], medicine [21], or real world agents [5], representations are sought that capture the qualitative behavior of the data. For the purposes of plotting [4], a primary concern is a reduction in the number of points. In other applications such as image processing [19,13], experimental sciences [21], computer science problems [9], and parameter estimation methods for ordinary differential equation (ODE) models [28], accurate representations of the data allow for more efficient and effective analysis.

Over the past 50 years, there has been considerable work on the problem of representing discrete data by a (piecewise) continuous function and the related problem of approximating one (piecewise) continuous function with another. In virtually all of this work, algorithms are presented that compute approximations that are best or at least good with respect to some metric and satisfy some list of constraints. The approximations are usually piecewise polynomials and often linear; in the latter case we call them piecewise linear representations (PLRs). The metric is generally either the $L_{2}$ norm (the least squares criterion) or the $L_{\infty}$ norm (the minimax criterion). Typically the constraint is the number of segments permitted. A complementary (and easier) problem that is frequently addressed is the determination of a piecewise poly-

*Received by the editors August 25, 2009; accepted for publication (in revised form) June 29, 2010; published electronically August 24, 2010.

http://www.siam.org/journals/sisc/32-5/76907.html

${ }^{\dagger}$ Department of Mathematics and Statistics, University of Guelph, Guelph, ON, N1G 2W1, Canada (eszusz@uoguelph.ca, AWillms@uoguelph.ca). This research was supported by the Natural Sciences and Engineering Research Council of Canada Alexander Graham Bell Canada Graduate scholarship. 
nomial approximation with the fewest number of segments given a maximum error tolerance. For either of these problems the approximating function may be discontinuous at the segment boundaries or constrained to be continuous, which is generally a more difficult case. The present work adds to this list by presenting an algorithm that approximates discrete data with a near minimax continuous piecewise linear function whose number of segments is not prespecified but rather determined by the constraint that either each interior segment must be at least some user-specified length, $t_{\min }$, or its slope must lie between its neighbors' slopes. Assuming that noise is random and equally likely to increase or decrease a recorded value, this constraint prevents the fitted curve from "inflecting" too much; that is, it prevents sign changes in the difference of slopes of consecutive segments from occurring too close together. It is a natural way of specifying a degree of smoothness. A constraint of this form makes the problem more difficult than simply finding an optimal solution for a given number of segments as we discuss in section 3.1.

1.1. Background. Some of the earliest work on data approximation was by Bellman [2] and Gluss [7] in 1961 and 1962, who described a dynamic programming solution to the problem of finding the best $\left(L_{2}\right.$ norm, but the $L_{\infty}$ norm could also be used) piecewise polynomial with $K$ pieces, fit to a given continuous function, $f(t)$. Bellman assumed the approximation was not continuous, while Gluss showed how to constrain the pieces so that their join points coincide with the original function. Their solution requires the computation of a function on an open interval, which is done by calculating this function on a prescribed partition. Hence the result is only as accurate as the spacing of the partition. Further, the algorithm is computationally intense requiring $O\left(N^{2} K\right)$ floating point operations, where $N$ is the number of points in the partition. The algorithm also works for the case of discrete data; in this case the solution is exact and the time required is $O\left(n^{2} K\right)$, where $n$ is the number of data points. Gluss also stated that the same procedure can be used to find the best continuous approximation where neighboring pieces match at the join points but need not coincide with the original function there, but this is not completely true as the problem becomes two dimensional. In particular, if $P_{k}\left(t_{a}, y_{a}, t_{b}, y_{b}\right)$ is the best (in the $L_{\infty}$ sense) continuous piecewise polynomial fit to $f(t)$ on $t_{a} \leq t \leq t_{b}$ with $k$ pieces that passes through $\left(t_{a}, y_{a}\right)$ and $\left(t_{b}, y_{b}\right)$, then the recursion is

$$
P_{k+1}\left(t_{0}, y_{0}, t, y\right)=\min _{\substack{t_{0} \leq \leq \leq t \\-\infty<z<\infty}} \max \left[P_{k}\left(t_{0}, y_{0}, s, z\right), P_{1}(s, z, t, y)\right]
$$

The approximations $P_{k}$ must be computed on some $N \times M$ grid, which requires $O\left(N^{2} M^{2} K\right)$ time. However, unlike the previous cases, if $f(t)$ is replaced with discrete data and the join points need not coincide with the original data, the solution still needs to be computed on a grid and the accuracy depends on the resolution.

In 1968 Phillips [17] gave an algorithm for finding a continuous PLR with the fewest line segments that lies within a specified deviation $\left(L_{\infty}\right.$ norm) to a continuous function whose second derivative is of constant sign. Wilson [29] first proposed in 1972 an algorithm to give a continuous or discontinuous PLR of discrete data that is within a given deviation and has a minimum number of segments. A year later, Douglas and Peucker [4] presented an efficient algorithm for reducing the number of points necessary to represent a curve given as a large, dense discrete data set, again using a specified error tolerance.

Also in 1972 Ramer [19] gave an iterative procedure for approximation of plane curves (open or closed) by a polygon with a small number of vertices. The fit criterion 
he used is the orthogonal distance to the line. A curve segment is first approximated by a straight line segment joining the first and last points. If the fit is not acceptable, then the segment is split at the point of maximum deviation. This idea of splitting at the point of maximum deviation is one we also employ; however, we do not anchor the two end points of every segment. His final product is a polygon (or polygonal line) of the $K$ connected termini. He gives the approximate complexity as between $O(n)$ and $O(n K)$ but typically $O(n \log K)$.

In a 1974 paper, Pavlidis and Horowitz [14] gave an algorithm for segmenting a set of points into a minimum (local, but they claim quite close to the global minimum) number of segments where in each segment the data are approximated by a polynomial of given degree under the constraint that the error bound (any norm) is below some given threshold. They focus on degree 1. Their approximations are, however, not continuous. In the same year Pavlidis and Maika [15] gave a functional iteration method for approximating a continuous function by polynomials on $K$ segments where the joins are not continuous. Basically, the segment boundaries are adjusted by some scaled portion of the difference in the error measures, since an optimal solution will have equal error on all segments; however, this would not be true for discrete data.

A method for fitting a Bézier curve to a digitized curve in the plane was presented by Schneider [20] in 1990. This technique is primarily useful for geometric curves in the plane as opposed to functions on a real line. It starts by fitting a single cubic Bézier curve to all the data, where the two exterior control points for the curve are fixed to be the first and last of the data points in the segment to be fit. The interior two control point locations are iteratively altered to reduce the orthogonal distance from points to the curve. If the fit is deemed insufficient, the segment is split at the point where the largest distance to the curve occurs and the resulting subsegments are fit, etc. The result is a curve that is continuous and passes through the end points of each segment.

A short review of various types of PLR algorithms can be found in the paper by Keogh et al. [9]. They categorized PLRs into three main approaches: (i) sliding window, in which data are processed from the first data point and segments are created as data are scanned; (ii) top-down, in which data are continually partitioned at the "best" locations into smaller segments until some stopping criterion is reached; and (iii) bottom-up, in which similar adjacent segments are merged, again until some stopping criterion is reached. They also introduced their own algorithm for online time series segmentation that was a combination of sliding window and bottom-up. Their algorithm requires $O\left(n^{2} / K\right)$ time [5]. Our algorithm could be classified as a topdown algorithm although we do not spend the time to determine the "best" partition point at each iteration, but rather partition at the point of largest error. This makes our algorithm considerably faster than the top-down algorithms described by Keogh et al. [9].

Using the $L_{2}$ norm, Palpanas et al. [12] have extended this work on online time series approximation giving an "amnesic" algorithm that permits greater error in approximating points from the past and approximates newly streamed points more accurately. The $L_{2}$ norm and the use of piecewise linear approximations allow them to compute the error of a merged segment from the errors of the two merging segments in constant time, and therefore their algorithm ends up requiring $O(n \log K)$ time if they use piecewise constant amnesic functions, longer if they use more complex amnesic functions. However, these results do not hold for the $L_{\infty}$ norm.

Monotone or quasi-monotone approximation is a related problem. Here, instead of fitting a parametric curve to the data, the goal is to segment the data into alternating 
quasi-monotonic increasing and decreasing segments. The "quasi" nature arises from allowing the data in an increasing (decreasing) segment to decrease (increase) by no more than some predefined distance $\delta$. In 2000 Stout [23] gave an optimal algorithm for unimodal regression, which has one increasing and one decreasing segment. His $L_{2}$ norm version requires $O(n)$ time while the $L_{1}$ version requires $O(n \log n)$. He did not use the $L_{\infty}$ norm. As a generalization of Stout's idea, Demetriou [3] provided software that produces a continuous piecewise monotonic approximation that is optimal in the $L_{2}$ norm and has a specified number, $K-1$, of "turns," that is, changes in the sign of the slope. The order is $O\left(n \sigma+K \sigma^{2}\right)$, where $\sigma$ is the number of local minima in the data, which is bounded by $n / 2$. Also recently, Lemire et al. $[10,5]$ provided an algorithm for optimal quasi-monotonic segmentation that takes $O(n \log n)$ time.

1.2. Motivation. In this paper we are interested in producing a continuous PLR with a minimal $L_{\infty}$ measure for use by algorithms that require the data to lie within some specified interval. In particular, parameter estimation methods for ODE models using interval analysis [8,11, 24, 25, 26] or monotonic discretizations [27] typically start with the assumption that the observed data lie within some known error bound. Further, we use a continuous PLR because the underlying function for which the data are a sampling is assumed to be continuous and we wish to use the PLR to construct a continuous band of constant height that contains the data. Good bounds for the data are an essential starting point for parameter identification techniques based on interval analysis [26] and monotonic discretizations [27]. A parameterized function (typically the solution of a system of ODEs) is assumed to represent the data. With these techniques one proves that provided the parameter values lie within some specified box in parameter space, then the function defined by these parameters yields values (intervals) that are within, intersect, or do not intersect prespecified intervals around the data. The determination of the boxes in parameter space is driven by the given data intervals; the smaller these intervals are, the more tightly one can constrain the parameter boxes. On the other hand, if the specified intervals are too small compared to the actual noise level in the data, then these techniques will reach the conclusion that no parameter values will allow the function to be consistent with the data. It is therefore necessary to provide intervals around the data that are large enough to account for noise but small enough to not lose the signal present in the data.

1.3. The problem. The mathematical problem for which the current algorithm provides an optimal or nearly optimal solution is as follows. We assume the data set $S=\left\{\left(t_{i}, y_{i}\right)\right\}_{i=1}^{n}$ is ordered so that the $t_{i}$ are increasing. For a set of join points, $\left\{\left(T_{j}, Y_{j}\right)\right\}_{j=1}^{N}$, the continuous piecewise linear curve through them is defined by

$$
M(t)=\frac{Y_{j+1}-Y_{j}}{T_{j+1}-T_{j}}\left(t-T_{j}\right)+Y_{j}, \quad T_{j} \leq t \leq T_{j+1}, \quad 1 \leq j \leq N .
$$

The $j$ th segment of $M(t)$ extends from $T_{j}$ to $T_{j+1}$ with length $L_{j}=T_{j+1}-T_{j}$ and slope $s_{j}=\left(Y_{j+1}-Y_{j}\right) / L_{j}$. Without loss of generality we assume $s_{j-1} \neq s_{j}, 1<j \leq N$; otherwise the join point $\left(T_{j}, Y_{j}\right)$ could be removed. Thus, at each point $T_{j}, 1<j<N$, the curve $M(t)$ has a slope discontinuity, which we label as positive if $s_{j-1}<s_{j}$ or negative otherwise. The optimization problem is the following.

Given $S$ and a user-specified value $t_{\min }$, choose $N$ and $\left\{\left(T_{j}, Y_{j}\right)\right\}_{j=1}^{N}$ to minimize

$$
h=2 \max _{1 \leq i \leq n}\left|y_{i}-M\left(t_{i}\right)\right|
$$


subject to

$$
\begin{aligned}
& \text { (i) } t_{1}=T_{1}<T_{2}<\cdots<T_{N-1}<T_{N}=t_{n} \quad \text { and } \\
& \text { (ii) } L_{j} \geq t_{\min } \quad \text { or } \quad s_{j-1}<s_{j}<s_{j+1} \quad \text { or } \quad s_{j-1}>s_{j}>s_{j+1}, \quad 1<j<N \text {. }
\end{aligned}
$$

In other words, we seek a curve $M(t)$ that is a minimax PLR of the data whose opposite-sign slope discontinuities are at least $t_{\min }$ apart.

The constraint given by (1.4) is novel. Virtually all algorithms described in the literature simply either place a constraint on the number of segments and attempt to minimize the error measure or place a constraint on the error and minimize the number of segments. Our constraint dictates that no segment whose slope is either below both its neighbors' slopes or above both its neighbors' slopes have horizontal length less than the user-specified value of $t_{\min }$. This is a natural constraint that prevents the PLR from following random noise too closely.

The solution to this optimization problem is not unique, but clearly always exists since a single straight line segment satisfies the constraints. This optimization problem is not easily solved by standard means since the constraints and the objective function are discontinuous with the variables $\left(T_{j}, Y_{j}\right)$ and since the number of variables is itself a variable to the problem. We were unable to get MATLAB's fmincon or fminimax routines to converge to a solution anywhere near as good as the solution obtained by our algorithm unless we gave our algorithm's solution to these MATLAB routines as an initial starting guess. We also coded a dynamic programming algorithm similar to those described by Gluss [7] in order to obtain an optimal solution confined to some prespecified $N \times M$ grid. This algorithm was prohibitively slow, $O\left(N^{2} M^{2} K\right)$, and its ability to find solutions better than our algorithm was contingent on making the grid sufficiently high in resolution. In contrast, the algorithm presented here always yields a solution satisfying the constraints and does so in $O(n K)$ time. Under some situations, described below, it may yield a band that is suboptimal, but of all of the experiments we have made so far, our algorithm yielded the optimal height more than half the time and the smallest value of the ratio of the optimal height to our height was 0.74 . Further, among the solutions with the same height $h$, this algorithm tends to select one that has smaller changes in the slope of the PLR at the joining points, that is, a relatively smoother solution.

Given the PLR obtained by the algorithm, a band of constant height $h$ that contains all the data is defined by $M(t) \pm h / 2$. This band is an effective representation of the data set that captures the directional trends of the data, gives a measure of the noise, and facilitates rapid interpolation of expected $y$ values at times between the recorded $t_{i}$ 's.

The second author recently presented an algorithm for bounding data with a piecewise linear band [28]; however, the current algorithm is an improvement on that one in several respects. The former algorithm was more heuristic in nature and did not produce bands that were solutions of any easily specified optimization problem. In addition, in order to produce satisfactory bands, the former algorithm allowed the user to specify a certain number $M$ of times where a segment with length less than $t_{\min }$ could have a slope smaller than both or larger than both its neighbors' slopes. There was a certain interplay between these two user-specified parameters and appropriate values were data-set dependent. In contrast, the current algorithm depends on just one user-specified parameter whose role is unambiguous. Finally, the former algorithm tended to produce bands that attenuated curves in the data. In particular, data points were clustered near the outside edge of the band at places 
where the band changed slope. The current algorithm produces bands where the data tend to be clustered near the center of the band even when the data points are curving upward or downward.

Section 2 of this paper describes the algorithm in detail, and section 3 gives the results of our algorithm acting on four different real time-series data sets and discusses the algorithm's optimality and scaling properties. Some concluding discussion is provided in section 4 .

2. The algorithm. The algorithm works on the data set in a top-down fashion by dividing it at certain data time points, into segments, acting on one segment at a time. Initially there is one segment encompassing the entire data set. Three actions are performed on a segment: sandwiching, splitting, and joining. Sandwiching a segment consists of determining the linear band of smallest vertical height that encompasses all the data in the segment. As described below, sandwiching also identifies an interior data time point, called the break point, at which to split the segment. A segment is split by defining two new segments from the original, one consisting of data points to the left of the break point and the other of those to the right. The break point belongs to both new segments. Joining neighboring segments consists of expanding both to the same height and then calculating the point where the segments intersect.

The algorithm operates in two modes: reduction and smoothing. In reduction mode, the algorithm iterates the sequence of splitting the tallest segment, sandwiching the two new segments, and joining them. The first iteration consists of only sandwiching the initial segment. The $i$ th iteration produces an intermediate solution with $i$ segments and band height $h_{i}$. It is possible that the joining operation causes the intermediate solution to violate (1.3); that is, a segment gets "swallowed." Such solutions are flagged but do not stop the algorithm since subsequent iterations may "unswallow" the segment. If (1.3) is satisfied by the intermediate solution, then this solution determines a maximal value $\tau_{i}$ such that if $t_{\min }$ is replaced by $\tau_{i}$ the constraint (1.4) is satisfied. The value $\tau_{i}$ is the length of the shortest segment between opposite-sign slope discontinuities, and may be infinity if no such constraining segment exists. Since the tallest segment is always split and since, as described below, the resulting two segments have height no larger than the original, the sequence of heights, $h_{i}$, is nonincreasing. However, the sequence $\tau_{i}$, although it tends to be flat or decreasing, occasionally also increases with $i$. The increases occur when splitting and sandwiching of a segment alters the joining points $T_{j}$ of a constraining segment in a way that increases its length. The iterations continue until the length of the constraining segment $\left(\tau_{i}\right)$ plus the lengths of the two neighboring segments is less than the user specified $t_{\min }$ value. Our experience is that this is sufficiently far to ensure that were the iterations to continue, subsequent $\tau_{i}$ values would not increase back up to $t_{\text {min }}$. The intermediate solutions are then checked to see which one having $\tau_{i} \geq t_{\min }$ and satisfying (1.3) has the minimal height. This is the candidate solution.

At this point the algorithm switches to smoothing mode, where it identifies segments of the candidate solution that when split and sandwiched will decrease changes in slope. Such segments are characterized by having a slope intermediate to its two neighbors and having a particular geometry of its enclosed data points as described below. Such splits are guaranteed never to cause a violation of (1.4) for the two new segments, but since their outside join points may alter, the neighboring two segments need to be checked against (1.3) and (1.4). Those splits that do not violate (1.3) and (1.4) are applied, and when all such splits have been made, the final solution is obtained. 
(a)

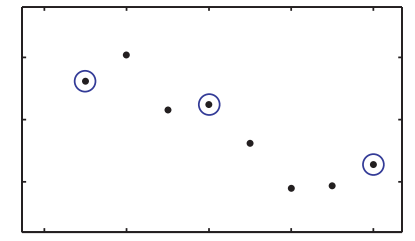

(d)

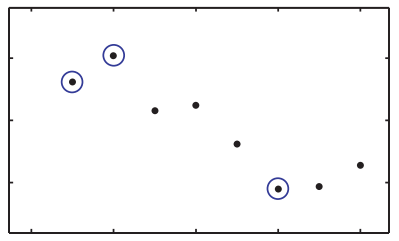

(b)

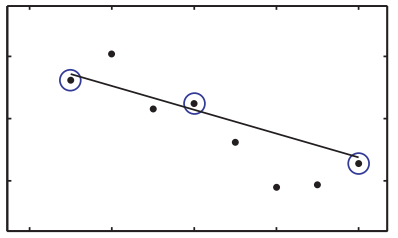

(e)

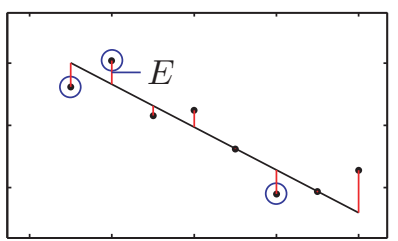

(c)

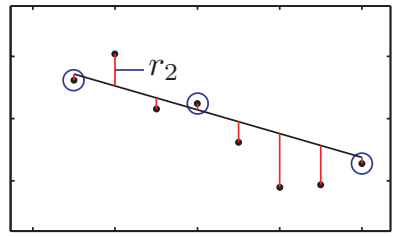

(f)

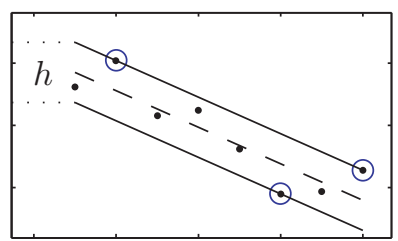

FIG. 2.1. Sandwiching a segment via the Remez exchange algorithm. (a) Segment data (in the $(t, y)$ plane) with three (circled) initial pivot points. (b) Unique line with an alternating distance of $\pm E$ from the pivots. (c) Residuals $r_{i}$ calculated for all data points. $r_{2}$ shown as an example. (d) Three new pivots assigned based on largest residuals of alternating sign. (e) New value for E and new line that is a distance $\pm E$ from pivots, and new residuals. (f) New pivots assigned, final best line (dashed), and final band of height $h=2|E|$ shown as solid lines.

2.1. Sandwiching. Let $f$ be a continuous function on $[a, b]$ and consider the infinity norm

$$
\|f\|_{\infty}=\max _{t \in[a, b]}|f(t)| .
$$

The Chebyshev equioscillation theorem $[1$, p. 224] states that for a given continuous function $f$ on $[a, b]$ and integer $n \geq 0$, there exists a unique polynomial, $p_{n}^{*}$, in the class $P_{n}$ of polynomials of degree less than or equal to $n$, such that

$$
E(f)=\left\|f-p_{n}^{*}\right\|_{\infty}=\min _{p_{n} \in P_{n}}\left\|f-p_{n}\right\|_{\infty} .
$$

Further, this polynomial is uniquely characterized by the fact that it has $n+2$ points, $t_{j}, 0 \leq j \leq n+1$, in $[a, b]$ satisfying $t_{0}<t_{1}<\cdots<t_{n+1}$ for which

$$
f\left(t_{j}\right)-p_{n}^{*}\left(t_{j}\right)=\sigma(-1)^{j} E(f),
$$

where $\sigma= \pm 1$, depending only on $f$ and $n$. The theorem applies to discrete data as well, in which case $[a, b]$ is replaced by a discrete set.

The Remez exchange algorithm is a well-known algorithm for finding the best (in the $L_{\infty}$ sense) approximating function in a Chebyshev space to some given function. The algorithm is described, for example, in Fraser [6], and proof of its convergence can be found in [22]. We use the Remez algorithm to find the minimizing first order polynomial for a given segment of data, that is, to sandwich it. The algorithm's time requirement is proportional to the number $m$ of data points in the segment. Relabel the data points in the segment as $\left\{\left(t_{i}, y_{i}\right)\right\}_{i=1}^{m}$. Essentially the algorithm finds the unique points $t_{i_{j}}, j=0,1,2$, which we call pivot points, by exchanging current candidates for points where $\left\|y_{i}-p_{1}\left(t_{i}\right)\right\|_{\infty}$ is larger. This procedure is illustrated in Figure 2.1. Upon a segment's creation, three of its data points are defined to be pivot points. For the initial segment, these are chosen to be the first, last, and middle data 


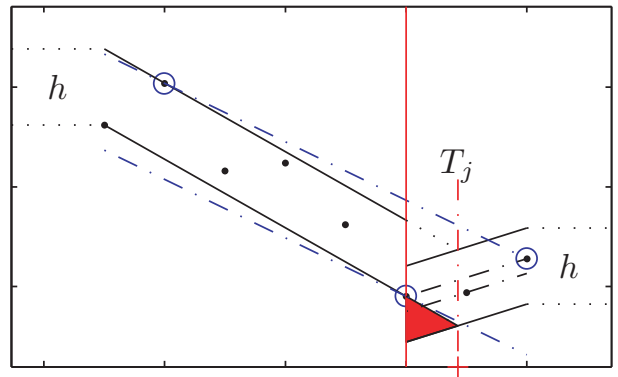

FIG. 2.2. The situation after the segment in Figure 2.1(f) has been split and both new segments sandwiched. The original unsplit segment along with its three pivot points (circled) are shown (dotted-and-dashed lines spanning entire data set). The right (jth) segment has been expanded to match the height of the left segment (assumed here to be the biggest of all segments). The original jth segment is also shown as dotted-and-dashed lines. The top and bottom lines of the left segment are extended (as dotted lines) to where it intersects the right segment at $T_{j}$. The shaded area indicates the part of the segment that gets removed.

points. For intermediate segments, which have just been split, the segment inherits two pivots from its parent segment and a third is selected (see below). The linear system

$$
y_{i_{j}}=a_{2} t_{i_{j}}+a_{1}+(-1)^{j} E, \quad j=0,1,2,
$$

is solved for $a_{1}, a_{2}$, and $E$, and the line $p_{1}(t)=a_{2} t+a_{1}$ is formed. The residuals $r_{i}=p_{1}\left(t_{i}\right)-y_{i}$ of each data point in the segment are determined. If no $r_{i}$ is greater in magnitude than $|E|$, we have our final solution, $p_{1}(t)$. If not, then new pivots are chosen by exchanging points with residuals larger than $|E|$ with the current pivots as described in Fraser [6], and the process is repeated. Typically, less than eight iterations are required. The band around this segment is then defined by $p_{1}(t) \pm|E|$. Pivot points lying on the lower boundary, $p_{1}(t)-|E|$, are called lower pivots, and those on upper boundary are upper pivots.

2.2. Splitting. Once a segment has been sandwiched, the middle pivot point is defined as the break point, for example, the lower circled point in Figure 2.1(f). When a segment is to be split, the split is made along the vertical line passing through the break point producing two new segments, one to the left of the break point and one to the right. The break point is the only common data point between the two segments. Each new segment inherits the break point and one of the two remaining pivot points. A third pivot point is chosen either at the opposite end from the break point or, if this is already a pivot, the middle point of the segment.

2.3. Joining. Since each segment gets sandwiched independently of its neighboring segments, each segment's height will be different and the ends of the bands around the segments will no longer match up at the break points, Figure 2.2. Let $h$ be the maximum height of all segments. To make a continuous band with constant height, all segments are first expanded vertically (equally up and down) to the height $h$ and then joining points, $T_{j}$, where the boundaries of the $j$ th segment intersect those of the $(j-1)$ st segment, are calculated, Figure 2.2. The join values depend only on the relative unexpanded heights of the segments and not the maximum height $h$. Let $h_{j-1}$ and $h_{j}$ be the heights before expanding and $s_{j-1}$ and $s_{j}$ be the slopes of segments $j-1$ and $j$, respectively. Let $\left(t_{p_{j-1}}, y_{p_{j-1}}\right)$ and $\left(t_{p_{j}}, y_{p_{j}}\right)$ be lower pivot points of the 
two segments. The equations for the bottom edge of the bands of two segments after they have both been expanded to height $h$ are

$$
\begin{aligned}
& y=y_{p_{j-1}}+s_{j-1}\left(t-t_{p_{j-1}}\right)+\frac{h_{j-1}-h}{2}, \\
& y=y_{p_{j}}+s_{j}\left(t-t_{p_{j}}\right)+\frac{h_{j}-h}{2} .
\end{aligned}
$$

The intersection of these two lines occurs at

$$
T_{j}=\frac{y_{p_{j}}-y_{p_{j-1}}+s_{j-1} t_{p_{j-1}}-s_{j} t_{p_{j}}-\frac{h_{j-1}}{2}+\frac{h_{j}}{2}}{s_{j-1}-s_{j}},
$$

which has no dependence on $h$. Equivalently, $T_{j}$ is the time point of intersection of the two midlines of the segment bands. Thus, we can calculate the join values at any stage of the algorithm and $T_{j}$ does not change unless segment $j-1$ or segment $j$ is sandwiched and split.

By joining segments as described above, it is clear that some area will be removed from part of a segment (the shaded area in Figure 2.2). However, for the following reason, this area will not contain any data points and thus may be safely discarded. When a segment is split, the slopes of the two new segments are bounded by the slope of the unsplit segment. Suppose the break point is a lower pivot point as in Figure 2.1(f). (The other case is analogous.) At any time after this segment is split in two, the segment immediately to the left of this break point will always have a slope smaller than the slope of the original unsplit segment. This is clearly true immediately after the first split since sandwiching pivots the band for the left segment clockwise, but it remains true even if subsequent splitting and sandwiching causes the slope of the segment immediately to the left of this break point to pivot back toward the original slope. Since the break point is a part of the segment immediately to its left, if that segment had a larger slope it would necessarily have a pivot point lying below the lower boundary of the original unsplit segment, which is a contradiction. Similarly, the slope of the segment immediately to the right of the break point will always have a slope larger than the slope of the original unsplit segment. Analogously, if the break point is an upper pivot point, then the slopes of the segments immediately to the left and right of the break point will always have slopes that are larger and smaller, respectively, than the slope of the unsplit segment.

This boundedness of the slopes of adjacent segments has two important consequences. First, once a split is made at a lower break point, the slope discontinuity at that point will always be positive. This property is called slope discontinuity sign preservation. Second, when rejoining is done for a lower break point, the extensions of the lower boundary of the segments on either side of the break point will necessarily lie in the area below the lower boundary of the original unsplit segment, Figure 2.2. The area being deleted lies below one of these extensions, hence is also in the region below the lower boundary of the original unsplit segment, and so contains no data.

2.4. Smoothing mode. Once a candidate solution is found, the algorithm refines the band by smoothing some of its edges around corners. Segments are checked left to right. Those segments whose slopes lie between their neighbors' slopes and whose break points are lower pivots if the slopes are increasing or upper pivots if the slopes are decreasing are split. Splitting such segments is guaranteed not to violate (1.4) for the new segments because the new segments will pivot in the direction toward the neighbor's slope, and this fact along with the slope discontinuity sign 
preservation property implies that both new segments will end up with slopes lying between their own neighbors' slopes. However, since splitting alters the adjacent $T_{j}$ values, the lengths of the two neighboring segments may change, and so (1.4) needs to be verified for them. If one of these fails this test or if (1.3) is violated for any of the altered segments, the split is undone and the algorithm moves to the next segment to the right. If a segment is split successfully, the new left segment is the next segment checked.

MATLAB code for this algorithm called TASLE (top-down algorithm splitting at largest error) is available on the second author's Web site: http://www.uoguelph. ca/ ${ }^{\sim}$ awillms/TASLE/.

3. Results. This section tests the algorithm on four different real time-series data sets. The first is echocardiogram (ECG) data from PhysioNet, with measurements in millivolts taken roughly every 0.008 seconds over a period of 5 seconds. The second data set is the average monthly exchange rate between the American and Canadian dollar from 1979 through 2009, obtained from the Pacific Exchange Rate Service. The third data set is the relative brightness of the variable star X Cygni recorded roughly once or twice per day over a five month period sourced from the American Association of Variable Star Observers. The fourth data set is voltage recordings from a pyloric dilator neuron of a spiny lobster, where the voltage in millivolts is measured regularly every 0.2 milliseconds for 2 seconds. These recordings were made by Jack Peck of Ithaca College. All of these data sets and detailed references to their original sources are available on the second author's Web site at http://www.uoguelph.ca/ awillms/TASLE/.

Various values of $t_{\min }$ were tested for each data set. Instead of the solution band changing with each new $t_{\min }$ tested, it was found that the solution remained unchanged as $t_{\min }$ varied over an interval and then suddenly changed as $t_{\min }$ passed a threshold value. This behavior is due to the fact that the data are discrete, and so small changes in $t_{\min }$ will make no difference in the band until $t_{\min }$ changes enough to cause a violation of the constraints. Figures 3.1-3.4 show the results of the four data sets tested at various values of $t_{\mathrm{min}}$.

The algorithm provided a rather nice result for the ECG data, Figure 3.1, since it is the timing and height of the peaks that are usually of the most importance, which are clearly produced by the band for $t_{\min }$ small enough $(\leq 0.016)$. For the exchange rate data, Figure 3.2, the algorithm was able to produce bands ranging from very small in height, which capture the small changes in the data, to large in height that only capture the general trends of the data. Similar results were obtained for the variable star data, Figure 3.3, and the neuron data, Figure 3.4. This variability in possible bands allows the algorithm to be used for applications ranging from those that may only need general trends to those that require a more detailed description of the data.

Smoothing had no effect on the first three data sets. This is likely due to the fact that all three data sets were quite variable and contained many sudden changes. It seems that smoothing is really only beneficial in data sets that tend to have relatively low amounts of noise and/or contain gradual changes in the data. Smoothing was only seen in the neuron data at the bottom of every trough. Figure 3.5 shows one of the troughs. As seen, the smoothing mode tends to soften some of the sharp corners by splitting segments at these locations and replacing them with two segments of intermediate slope. 
(a)

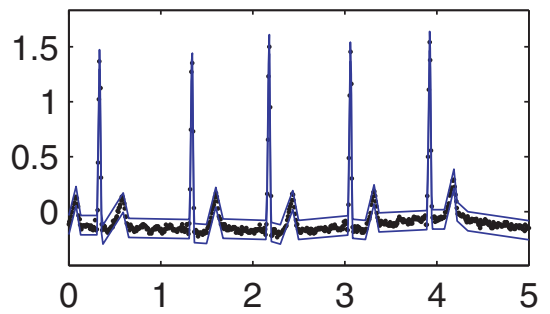

(c)

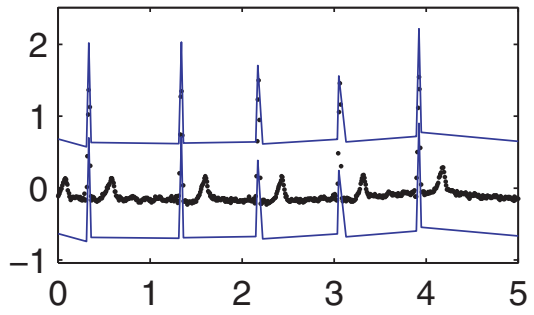

(b)

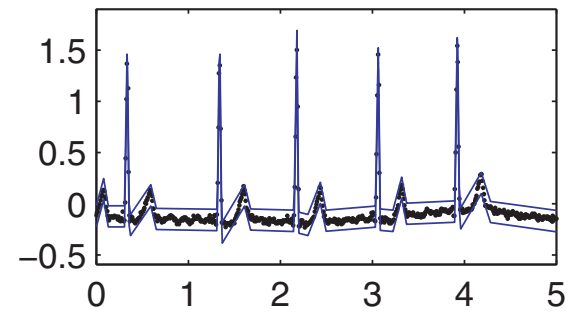

(d)

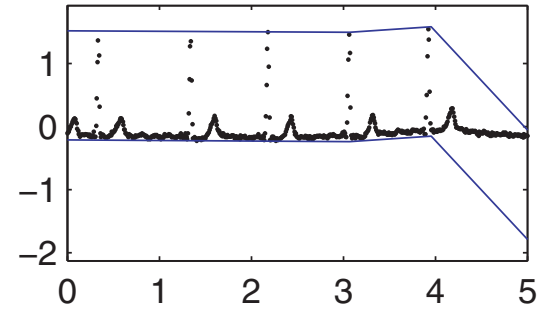

FIG. 3.1. ECG data enclosed by four different bands. Horizontal axis is in seconds, and vertical axis is in millivolts. $t_{\min }$ ranges are accurate to three decimal places. The corresponding heights are given. (a) $t_{\min }=0.007$, height $=0.1748$; (b) $t_{\min } \in[0.008,0.015]$, height $=0.2075$; (c) $t_{\min }=0.016$, height $=1.3177$; and (d) $t_{\min } \geq 0.017$, height $=1.7313$.

(a)

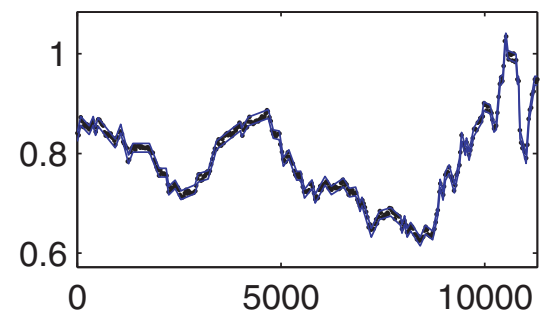

(c)

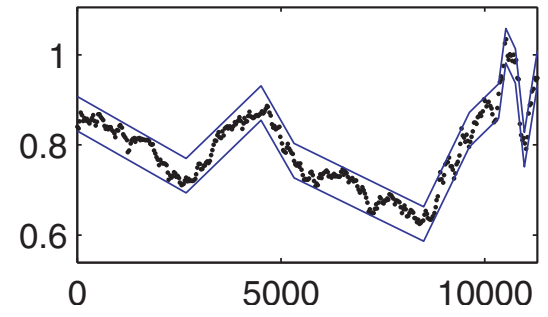

(b)

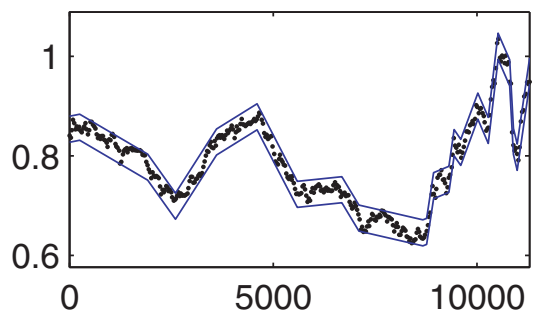

(d)

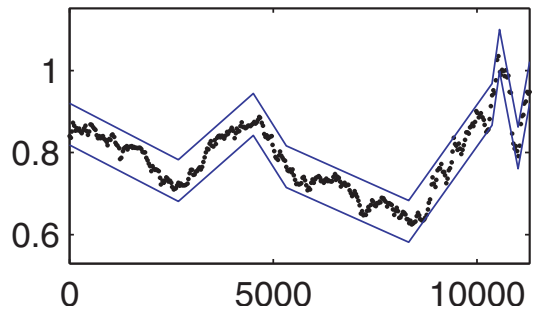

FIG. 3.2. Monthly exchange rate data between the American and Canadian dollar enclosed by four different bands. Horizontal axis is in days, and vertical axis is in U.S. dollars. $t_{\min }$ ranges are accurate to one decimal place. The corresponding heights are given. (a) $t_{\min } \in[28.1,29.6]$, height $=0.0173$; (b) $t_{\min } \in[29.7,89.7]$, height $=0.0523$; (c) $t_{\min } \in[89.8,172.7]$, height $=0.0763$; and (d) $t_{\min } \geq 172.8$, height $=0.1017$. 
(a)

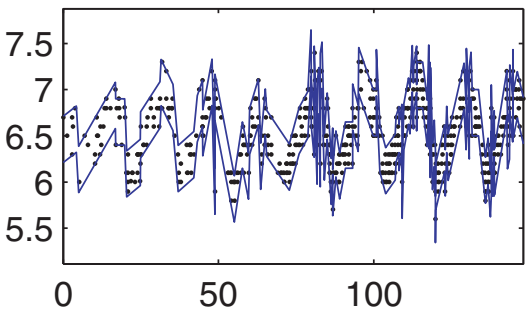

(c)

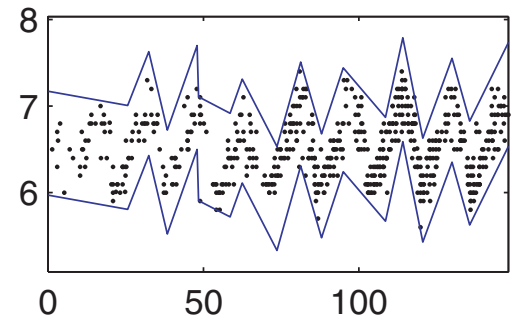

(b)

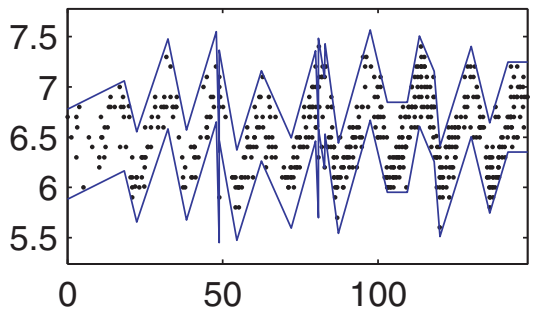

(d)

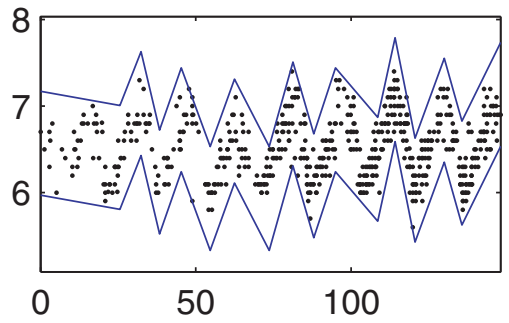

Fig. 3.3. Variable star data for the star X-Cygni from April 29, 2009, to September 25, 2009. Horizontal axis is in days, and vertical axis is in relative brightness. $t_{\min }$ ranges are accurate to four decimal places. The corresponding heights are given. (a) $t_{\min } \in[0.0004,0.0011]$, height $=0.5000$; (b) $t_{\min } \in[0.0012,0.0038]$, height $=0.8973 ;$ (c) $t_{\min } \in[0.0039,0.4636]$, height $=1.2001$; and (d) $t_{\min } \geq 0.4637$, height $=1.2004$.

(a)

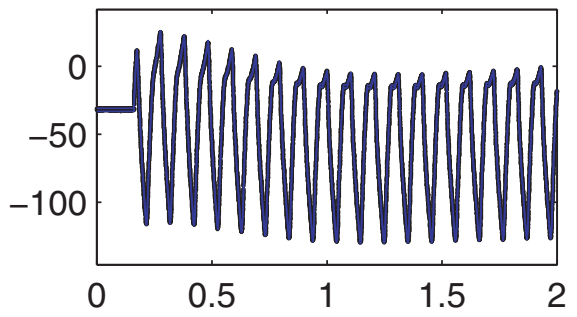

(c)

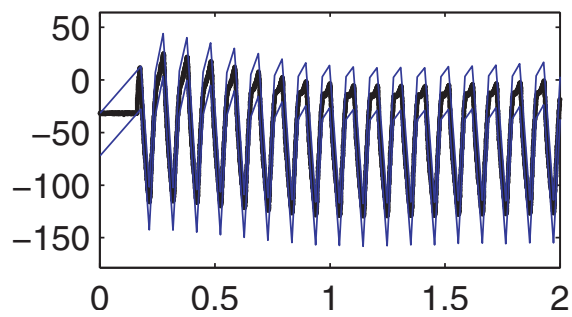

(b)

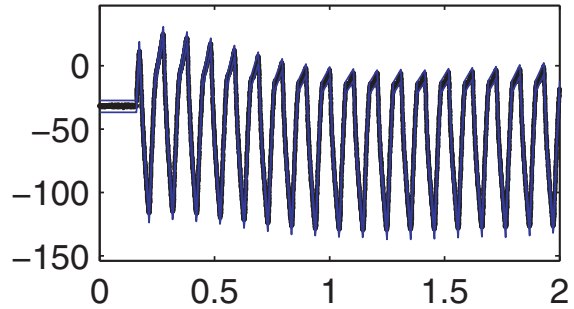

(d)

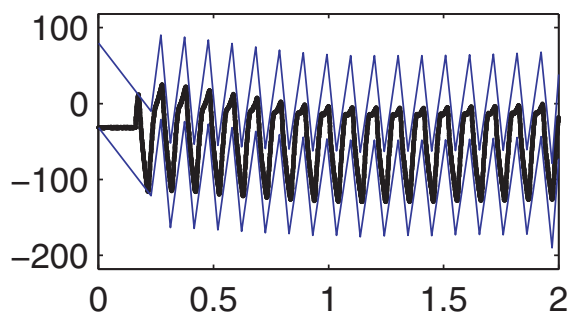

Fig. 3.4. Current-clamp data from the pyloric dilator neuron. Horizontal axis is in seconds, and vertical axis is in millivolts. $t_{\mathrm{min}}$ ranges are accurate to four decimal places. The corresponding heights are given. (a) $t_{\min } \in[0.0006,0.0017]$, height $=2.1875$; (b) $t_{\min } \in[0.0094,0.0118]$, height $=$ 9.3435; (c) $t_{\min } \in[0.0127,0.0257]$, height $=40.6393$; and (d) $t_{\min } \in[0.0323,0.0412]$, height $=$ 111.3263 . 


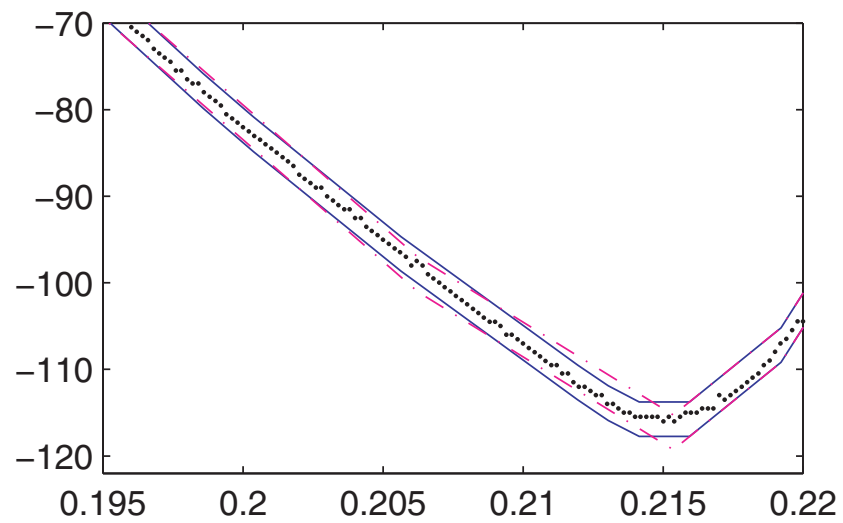

FIG. 3.5. Solution $(0.195 \leq t \leq 0.22)$ for the current-clamp neuron data when $t_{\min }=0.003$ with (solid lines) and without (dashed lines) the smoothing mode.

3.1. Optimality and speed. As mentioned in section 1.3, the optimization problem is not amenable to standard procedures. We coded the optimization problem for MATLAB's fmincon and fminimax functions using a fixed number of segments in order to keep the number of variables a constant. When we provided them with our algorithm's solution as an initial starting point, they were able to converge to a solution. We also implemented a dynamic programming solution to the problem. This procedure computes the best PLR on a predefined $N \times M$ grid; that is, the join points are constrained to lie on one of the grid points. Although prohibitively slow, $O\left(N^{2} M^{2} K\right)$, this algorithm is guaranteed to produce the optimal solution with segment ends constrained to the lie on the grid.

The solutions obtained from the MATLAB routines and those obtained by the dynamic programming algorithm were either exactly equal to those obtained from our algorithm or a little better. Improvement occurred when the constraining segment for our solution (the segment with the largest height) had a pivot point near the edge of the segment and the joining value for this segment and the neighboring segment ended up farther inside the constraining segment than the pivot point. Thus, after joining, this pivot point actually resided in the neighboring segment and consequently was no longer a constraint on the original segment. In this situation fmincon was able to reduce the height of the constraining segment a little bit by mildly shifting a few of the neighboring $\left(T_{j}, Y_{j}\right)$ points. The dynamic programming algorithm was also able to find a better solution if the grid was sufficiently refined. The improvement, however, was generally mild. In all such examples that we came across, the improvement in our algorithm's height was less than $26 \%$.

Although our algorithm does not in general find the optimal solution, comparison with the guaranteed optimal solution obtained from the dynamic programming algorithm applied to a very fine grid illustrates that our algorithm, at least in the examples we have tested, finds a solution that is close to the global optimum. This is not surprising, since by construction our solution has a constraining segment where the band obtained by the sandwiching procedure is optimal. In order to reduce $h$, this segment must be reduced; however, our algorithm would have attempted to split this segment and rejected it as violating constraints. Thus the only way to reduce $h$ would be to shift some data between segments, either shifting data out of the con- 


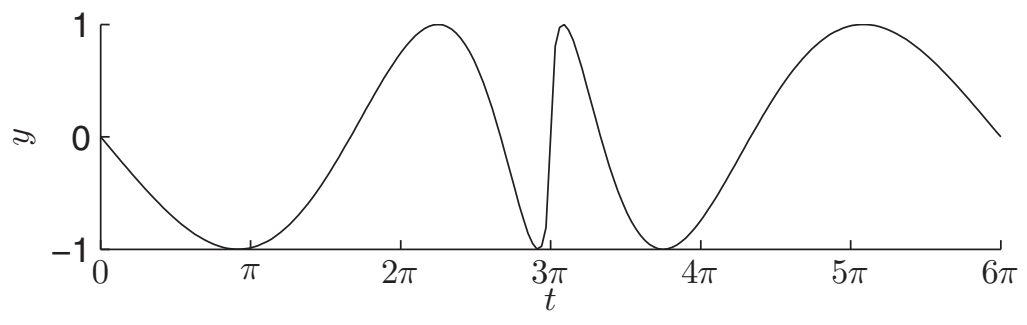

FIG. 3.6. Function sampled for timing experiments.

straining segment or splitting it and shifting data into it from neighboring segments. Our algorithm does not permit such shifting for reasons discussed in section 4 .

Our algorithm scales linearly with the number of data points. Splitting and joining segments requires constant time, and sandwiching a segment with $N$ points via the Remez algorithm requires $O(N)$ time. If $K$ is the number of segments and $n$ the total number of data points, then, since each segment can have at most $n$ points, the time required is bounded above by $O(n K)$. However, typically the size of the segments is reduced as more segments are split. Assuming optimistically that when segments are split they are split in half, then the segments that were sandwiched will have had approximately the following length distribution: 1 of length $n, 2$ of length $n / 2,4$ of length $n / 4$, etc. In this case the total time for the algorithm would be $O(n \log K)$. This estimate is better than can be expected in reality since segments will not always split near their middles, but will often split near their edges. Therefore the time required is bounded above by $O(n K)$ but may often be closer to $O(n \log K)$.

Timing experiments were performed on a $3.06 \mathrm{GHz}$ Pentium 4 machine using data $\left(t_{i}, y_{i}\right)$ generated from the expressions

$$
\begin{aligned}
t_{i} & =i \frac{6 \pi}{\rho}, \quad 0 \leq i \leq m \rho, \\
\theta_{i} & =\frac{t_{i} \quad \bmod 6 \pi}{3 \pi}-1, \\
y_{i} & =0.05 \epsilon_{i}+\sin \left(3 \pi \operatorname{sgn}\left(\theta_{i}\right) \sqrt{\left|\theta_{i}\right|}\right),
\end{aligned}
$$

where $\epsilon_{i}$ is a normally distributed random variable with mean zero and variance one, $m$ is an integer in the range $[1,32]$, and $\rho$ is a value in $\{100,200,400,800\}$. These data are a noisy sampling of $m$ periods of the function shown in Figure 3.6. As can be seen, this function has both slow and fast oscillations. The value $\rho$ controls the density of the sampling. When $m=32$ and $\rho=800$, the data set has 25,600 points. For each value of $m$ and $\rho$ selected, 50 instances of the data were generated and PLRs were obtained from the algorithm using $t_{\min }=\pi / 16$. The algorithm was coded in MATLAB for ease; however, greater efficiency could be obtained by coding in $\mathrm{C}$ and optimizing the implementation for speed. Figure 3.7 displays the results of these timing experiments where the number of cpu seconds required for the algorithm to complete is plotted against the product of the number of data points $n$ and the number of segments $K$ in the PLR. For fixed values of $m$ and $\rho, n$ is fixed but $K$ may vary since the particular instance of generated data will affect the number of segments in the final PLR, although typically $K$ will not vary too much. For this reason, the 50 instances for a given set of $m$ and $\rho$ values form clusters of 50 points in the plots in this figure. Several facts are evident from this figure. First, for each density level 
(a)

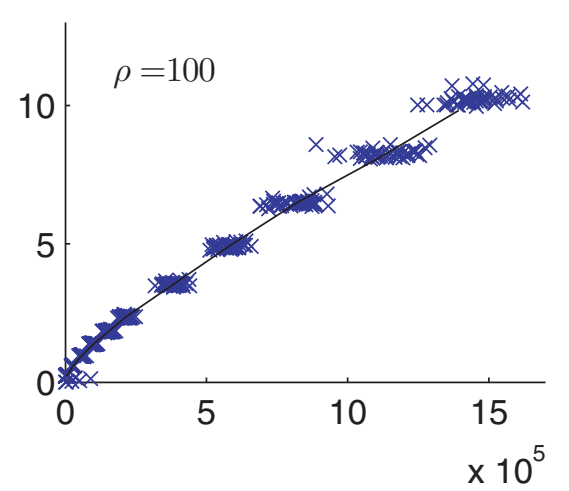

(c)

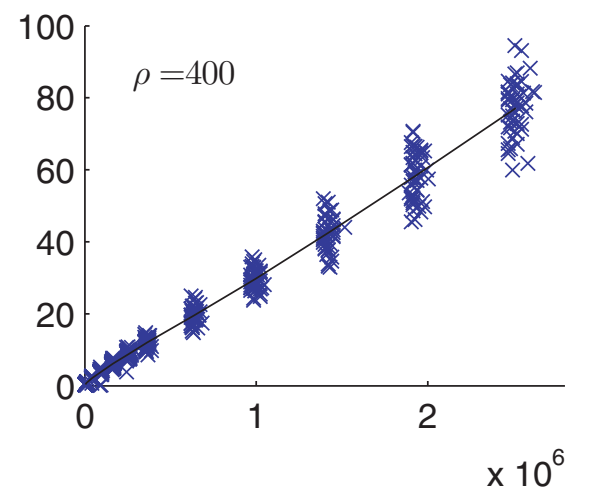

(b)

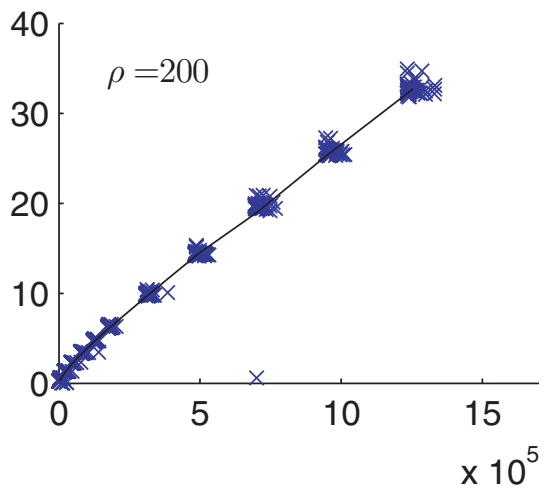

(d)

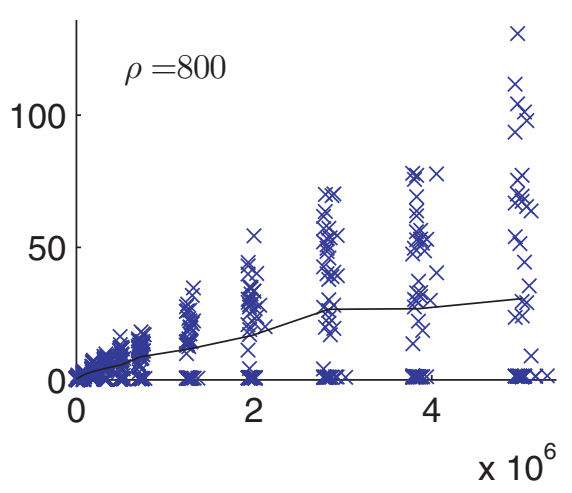

FIG. 3.7. Time required for the algorithm to obtain a PLR for the data given by (3.1)-(3.3) for various values of $m$ and four different density values $\rho$ : (a) 100, (b) 200, (c) 400, (d) 800. The vertical axis is cpu seconds, and the horizontal axis is the product $n K$. The solid lines are averages for each density level. Note that scales vary on these plots.

$\rho$, the time required clearly has a straight line upper bound as $n K$ gets large, and the average time is also well approximated by a straight line, illustrating the $O(n K)$ scaling. Second, at low density, Figure 3.7a, but not at higher densities, the time required does not vary substantially with $K$, evidenced by the fact that each cluster of 50 shows very little vertical variation. Third, the algorithm is fastest at the lowest density investigated, Figure $3.7 \mathrm{a}$, where the points all lie roughly on a line of slope 8 cpu seconds per million $n K$, compared to about a slope of 30 for the upper bounds at the higher densities. Finally, the variation in time required increases significantly as the density increases. In particular, there are a substantial number of trials at the highest density, Figure 3.7d, where the time required was very small. It appears that the algorithm scales best at relatively low densities, but at very high densities the algorithm will often outperform even the low density case.

4. Discussion. The algorithm described here rapidly produces a piecewise linear band of constant height centered around a given time series data set. The band is both a feasible and an optimal or nearly optimal solution of the constrained optimization problem given in section 1. Unlike the algorithm introduced in [28], the band constructed by this algorithm does not produce boundaries that hug outside curves 
of the data. Instead, the data points tend to be clustered near the center of the band. The "smoothness" of the resulting band is controlled by the user-defined parameter $t_{\text {min }}$, which provides a lower bound for the length of segments whose slopes do not lie between their neighbors' slopes.

If the solution produced by the algorithm is suboptimal, it appears to be only mildly so. In all of the examples we have tested, either we have not been able to find a better solution or the improvement in the height has been less than $26 \%$. As noted in the previous section, once our algorithm determines that subsequent splitting of the constraining (largest height) segment would yield length constraint violations, it is impossible to further reduce the height without shifting some data between segments. However, in general it is not possible to do so with our algorithm for the following reason. When a segment is to be split, a break point is identified in the interior of the segment. If the break point is on the lower boundary, then after splitting the slope of the new segment to its left will be smaller than the slope of the unsplit segment, and the slope of the new segment to its right will be larger than the slope of the unsplit segment (and vice versa if the break point is on the upper boundary). Subsequent splitting of these new segments will alter the slopes of the segments abutting the original break point. However, as shown in section 2.3, since the original break point remains part of both abutting segments, this relationship between the slope of the unsplit segment and the slope of the abutting segments remains. This relationship is essential when proving that no data are lost outside the band when neighboring segments are joined. Thus if data are shifted from one segment to another, then in general it is possible to lose data when the segments are joined into a single band.

Our algorithm remains in reduction mode (splitting the largest height segment, sandwiching the two new ones, and joining them with their neighbors) until the length of the constraining segment $\left(\tau_{i}\right)$ plus the lengths of the two neighboring segments is less than the user specified $t_{\min }$ value. In our experience this is more than sufficient to ensure that subsequent iterations would not produce $\tau$ values larger than $t_{\text {min }}$. However, this is just a heuristic, and one could easily alter this criterion. In the extreme, one could continue in reduction mode until the height is zero (the segments each contain two data points and the resulting band is simply a collection of line segments connecting the data); however, this tends to substantially lengthen the time required for the algorithm to complete without gain since in a typical situation $t_{\text {min }}$ will be large enough to contain many data points and computing solutions where most of the segments have much shorter lengths is of no value.

The smoothing mode in our algorithm typically produces only mild changes to the solution. As illustrated in Figure 3.5, the smoothing mode tends to soften some of the sharp corners by splitting segments at these locations and replacing them with two segments of intermediate slope.

The representation of the data provided by this algorithm is useful in a number of applications. Parameter identification schemes for ODEs based on interval analysis $[8,11,24,25,26]$ or monotonic discretizations [27] start with the assumption that the data points lie in some interval as do various methods for fitting curves to data $[16,18]$. However, the data are usually available as a set of discrete points, and so determining appropriate intervals is a necessary first step. Simply adding some measure of the noise $\epsilon$ to the data, $y_{i} \rightarrow y_{i} \pm \epsilon$, yields intervals that are as noisy as the data. The algorithm presented here yields intervals that essentially smooth some of the noise. In addition, not only are intervals provided at the recording times, $t_{i}$, but also at any time value. This representation is therefore especially suitable for schemes that 


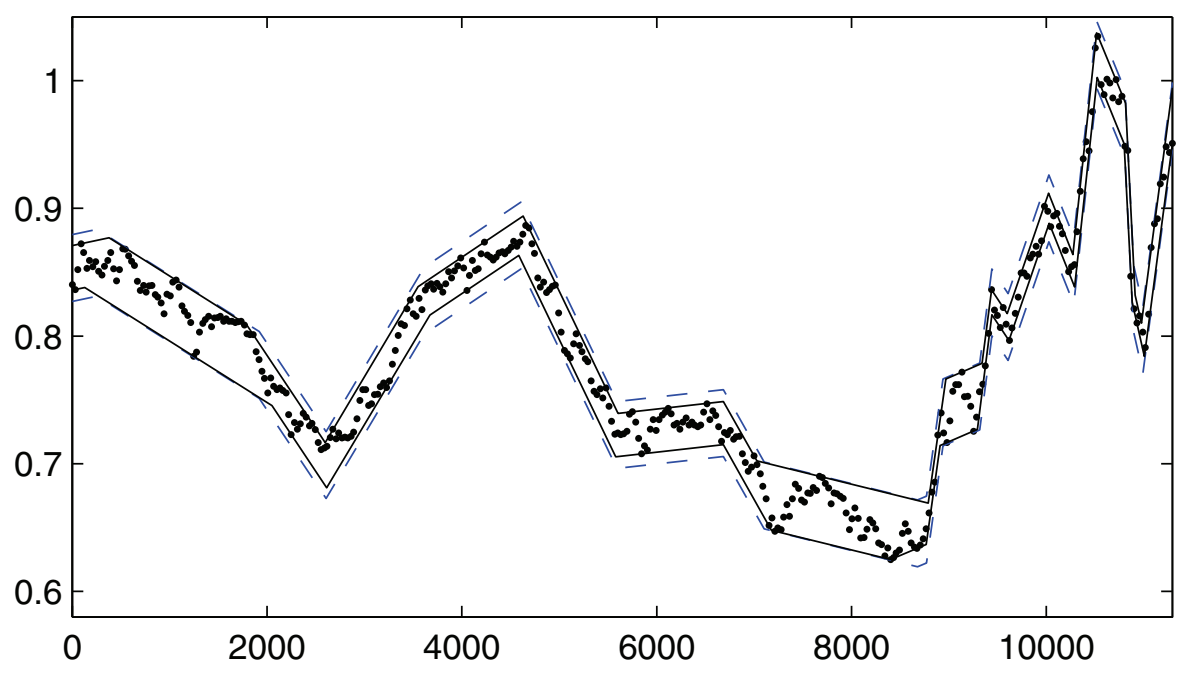

FIG. 4.1. Variable height band for U.S.-Canada exchange rate data with $t_{\min }=40$. Solid line is the variable height band, and dotted line is the band as in Figure 3.2(b).

need to interpolate the recorded data and establish intervals at the interpolated time points.

One generalization of the algorithm would be to allow a continuous band that does not have constant height. One way of producing such a band would be to run the current algorithm but alter the joining operation. Currently, the segments are widened to the constraining height and then joined, which results in intersections of the upper and lower boundaries of the band segments at the same time value. Instead, the segments could retain their own heights, and the upper and lower boundaries could be joined with their neighbors independently. A typical result is displayed in Figure 4.1 where we have fit the U.S.-Canada exchange rate data in such a manner. Since the upper and lower boundaries of a segment intersect with its neighbors' at different time values, the length of the segment needs a more precise definition in order to apply the constraints (1.3) and (1.4). One possible way to define the ends of a segment would be to determine where the midline of the segment intersects its neighbors' midlines. This definition yields lengths that are identical to that used in the current algorithm. However, under this definition it is possible that one boundary (the lower or the upper) of a segment could be swallowed by its neighbors without violating the constraint (1.3) since the midline length may still be positive. In this case, the join point for such a boundary segment could be replaced by the intersection of the left and right neighbors' boundaries. The advantage of this definition is that the current algorithm only requires an alteration in the final calculation of the output points $\left(T_{j}, Y_{j}\right)$; the main loop and stopping criteria remain unchanged. Another reasonable choice for the definition of the length of the segment would be to take the minimum horizontal distance between either of the join points on the left and either of those on the right. This definition would prevent swallowing of one boundary of a segment, but would require more changes to the current algorithm to implement. In either case, if there are significant noise level variations, focussing on splitting the tallest segment will result in segments in low noise areas being less optimal than in high noise areas. In order to obtain a solution that has tight segments in areas of low 
noise, it would be necessary for the algorithm to abandon splitting of large segments and focus on smaller ones prior to when the solution constraining length $\tau_{i}$ becomes smaller than $t_{\min }$. This would require further changes to the current algorithm.

Acknowledgments. We would like to thank the reviewers for their helpful comments.

\section{REFERENCES}

[1] K. E. Atkinson, An Introduction to Numerical Analysis, 2nd ed., John Wiley \& Sons, New York, 1989.

[2] R. Bellman, On the approximation of curves by line segments using dynamic programming, Commun. ACM, 4 (1961), p. 284.

[3] I. C. Demetriou, Algorithm 863: L2WPMA, a Fortran 77 package for weighted least squares piecewise monotonic data approximation, ACM Trans. Math. Software, 33 (2007), Article 6.

[4] D. H. Douglas And T. K. Peucker, Algorithms for the reduction of the number of points required to represent a digitized line or its caricature, Canad. Cartographer, 10 (1973), pp. $121-122$.

[5] W. Fitzgerald, D. Lemire, And M. Brooks, Quasi-monotonic segmentation of state variable behavior for reactive control, in Proceedings of the National Conference on Artificial Intelligence, Vol. 20, Part 3, 2005, pp. 1145-1150.

[6] W. FRASER, A survey of methods of computing minimax and near-minimax polynomial approximations for functions of a single independent variable, J. ACM, 12 (1965), pp. 295-314.

[7] B. GLuss, Further remarks on line segment curve-fitting using dynamic programming, Commun. ACM, 5 (1962), pp. 441-443.

[8] L. JAULIN, Nonlinear bounded-error state estimation of continuous-time systems, Automatica J. IFAC, 38 (2002), pp. 1079-1082.

[9] E. Keogh, S. Chu, D. Hart, And M. Pazzani, An online algorithm for segmenting time series, in Proceedings of the IEEE International Conference on Data Mining, San Jose, CA, 2001, pp. 289-296.

[10] D. Lemire, M. Brooks, And Y. YAn, An optimal linear time algorithm for quasi-monotonic segmentation, Int. J. Comput. Math., 86 (2009), pp. 1093-1104.

[11] Y. LIN AND M. A. STADTHERR, Guaranteed state and parameter estimation for nonlinear continuous-time systems with bounded-error measurements, Ind. Eng. Chem. Res., 46 (2007), pp. 7198-7207.

[12] T. Palpanas, M. Vlachos, E. J. Keogh, D. Gunopulos, and W. Truppel, Online amnesic approximation of streaming time series, in ICDE, IEEE Computer Society, Washington, DC, 2004, pp. 338-349.

[13] T. PAVLIDIS, Waveform segmentation through functional approximation, IEEE Trans. Comput., C-22 (1973), pp. 689-697.

[14] T. Pavlidis and S. L. Horowitz, Segmentation of plane curves, IEEE Trans. Comput., C-23 (1974), pp. 860-870.

[15] T. PAVlidis AND A. P. MAIKA, Uniform piecewise polynomial approximation with variable joints, J. Approx. Theory, 12 (1974), pp. 61-69.

[16] R. K. Pearson, Fitting straight lines to poor quality $(x, y)$ data, Math. Comput. Modelling, 16 (1992), pp. 71-80.

[17] G. M. PhilliPs, Algorithms for piecewise straight line approximations, Computer J., 11 (1968), pp. $211-212$.

[18] H. Piet-Lahanier And E. Walter, Exact recursive characterization of feasible parameter sets in the linear case, Math. Comput. Simulation, 32 (1990), pp. 495-504.

[19] U. RAmer, An iterative procedure for the polygonal approximation of plane curves, Comput. Graphics Image Processing, 1 (1972), pp. 244-256.

[20] P. J. SchneIDER, An algorithm for automatically fitting digitized curves, in Graphics Gems, A. Glassner, ed., Academic Press, Boston, MA, 1990, pp. 612-626.

[21] H. Shatkay And S. B. Zdonik, Approximate queries and representations for large data sequences, in Proceedings of the 12th IEEE International Conference on Data Engineering, 1996, pp. 536-545.

[22] E. L. Stiefel, Numerical methods of Tchebycheff approximation, in On Numerical Approximation, R. E. Langer, ed., University of Wisconsin Press, Madison, WI, 1959, pp. 217-232.

[23] Q. F. Stout, Optimal algorithms for unimodal regression, Comput. Sci. Statist., 32 (2000), pp. $1-8$. 
[24] W. Tucker, Z. Kutalik, And V. Moulton, Estimating parameters for generalized mass action models using constraint propagation, Math. Biosci., 208 (2007), pp. 607-620.

[25] W. Tucker And V. Moulton, Parameter reconstruction for biochemical networks using interval analysis, Reliab. Comput., 12 (2006), pp. 389-402.

[26] E. Walter AND M. KIEFFER, Guaranteed nonlinear parameter estimation in knowledge-based models, J. Comput. Appl. Math., 199 (2007), pp. 277-285.

[27] A. R. Willms, Parameter range reduction for ODE models using cumulative backward differentiation formulas, J. Comput. Appl. Math., 203 (2007), pp. 87-102.

[28] A. R. Willms, Bounding data with a piecewise linear band, SIAM J. Sci. Comput., 31 (2009), pp. 2361-2367.

[29] D. G. Wilson, Piecewise linear approximations of fewest line segments, in AFIPS Conference Proceedings Vol. 40, the 1972 Spring Joint Computer Conference, Montvale, NJ, 1972, pp. 187-198. 